\title{
The association between reported nightmares and depressive symptoms in a non-clinical large sample of Arab students
}

\begin{abstract}
Background: Research studies on sleep medicine and disorders using Arab participants are limited, particularly the relationship between nightmares and psychopathology. Cross-cultural studies may help to better understand this association.
\end{abstract}

Objective: The present study aimed to explore the correlation between reported nightmare and depressive symptoms in an under-studied sample of Arab students.

Methods: A convenience non-clinical sample of 8, 218 school and university Arab students was recruited. They were 13 age groups from 12 to 24 years. The self-rating scale of nightmares was assessed based on the participant's perceived frequency during the last month. Likert-type response was used as follows: 0 (No), 1 (A little), 2 (Moderate), 3 (Much), and 4 (Very much). The Center for Epidemiological Studiesdepression Scale was used.

Results: All the Pearson correlations between reported nightmares and depressive symptoms were statistically significant and positive in all the 13 age groups and ranged from 0.295 to 0.619 with a median of 0.402 .

Conclusion: based on a large sample of Arab students, and consistent with Western findings, the experience of nightmares associated with depressive symptoms. Future research would investigate their co-morbidity in a clinical sample.

Keywords: nightmares, depressive symptoms, arab students, mental disorders, psychopathology
Volume I Issue 2- 2017

\author{
Abdel Khalek AM \\ Department of Psychology, University of Alexandria, Egypt
}

Correspondence: Ahmed M.Abdel-Khalek, Department of Psychology, Faculty of Arts, University of Alexandria, Egypt. Email aabdel-Khalek@hotmail.com

Received: July 30, 2017 | Published: October 27, 2017

\section{Introduction}

Sleep disorder studies using Arab participants are underrepresented in the field of sleep medicine and psychopathology. However, three articles were published on nightmares with Arab samples. ${ }^{1-3}$ The last paper studied the association between reported nightmares and anxiety. Given the specific similarities and differences between anxiety and depression, it is important to study the nightmare-depression association. Because this association may change from country to another, cross-cultural investigations are needed. Therefore, the present investigation aimed to explore the relationship between reported nightmares and depressive symptoms in a cross-sectional non-clinical sample of Arab children, adolescents, and adults. The International Classification of Sleep Disorders (ICSD-R; American Academy of Sleep Medicine) ${ }^{4}$ identified more than 90 sleep pathologies, one of them is nightmares. The fifth edition of the Diagnostic and Statistical Manual of Mental Disorders (DSM 5; American Psychiatric Association) $)^{5}$ defined the nightmares as "typically lengthy, elaborate, story like sequences of dream imagery that seem real and that incite anxiety, fear, or other dysphoric emotions. Nightmare content typically focuses on attempts to avoid or cope with imminent danger but may involve themes that evoke other negative emotions... On awakening, nightmares are well remembered and can be described in detail... Nightmares usually terminate with awakening and rapid return of full awareness. However, the dysphoric emotions may persist into wakefulness and contribute to difficulty returning to sleep and lasting daytime distress". A continuum exists between bad dreams (without awakening) and nightmare (with awakening), with nightmares exhibiting the strongest correlation to psychopathology measures. ${ }^{6}$

Nightmares occur out of REM sleep in the form of terrifying dreams, usually in the middle or last part of the night. They are remembered by the individual, who usually wakes up suddenly with minor autonomic changes, in contrast to the distinctive and disturbing dream content. Nightmares usually start as early as age 3 and generally decrease in frequency and intensity over successive decades. Twinbased studies disclosed some genetic effects on the distribution for age and co-occurrence with other parasomnias. ${ }^{7}$ Nightmare disorder is listed as a distinct disorder in the International Classification of Sleep Disorders (American Academy of Sleep Medicine) ${ }^{4}$ the DSM 5 (American Psychiatric Association), ${ }^{5}$ and the International Statistical Classification of Diseases and Related Health Problems (ICD-10). ${ }^{8}$ Although nightmares are officially listed as sleep disorders, they should not necessarily be considered disorders requiring treatment, for two reasons as stated by Hartman. ${ }^{9}$ First, having some nightmares is entirely normal. Over 50 per cent of children, and perhaps all children aged 3, 4, and 5 years have nightmares; even among college students, most report occasional nightmares. Second, among subjects reporting a lifelong history of frequent nightmares, over half expressed no desire to get rid of the nightmares. Some of the subjects were artists and actually made use of the nightmares in their work; 
others felt the nightmares were a part of their personality, which they did not want, altered. In the present author's view, if the nightmare causes clinically significant distress or impairment in important areas of functioning, it must be considered a disorder requiring intervention. The most common causes of nightmares are as follows: sleep deprivation causing REM sleep rebound, narcolepsy, REM sleep behavior disorder, schizophrenia and schizoid personalities, anxiety states, drugs, e.g., L-dopa, beta blockers, and drug withdrawal, e.g., antidepressants, alcohol. ${ }^{10}$

The DSM 5 indicated that "prevalence of nightmares increases through childhood into adolescence. From $1.3 \%$ to $3.9 \%$ of parents report that their preschool children have nightmares "often" or "always". Prevalence increases from ages 10 to 13 for both males and females but continues to increase to ages 20-29 for females (while decreasing for males), when it can be twice as high for females as for males. Prevalence decreases steadily with age for both sexes, but the gender difference remains. Among adults, prevalence of nightmares at least monthly is $6 \%$, whereas prevalence for frequent nightmares is $1 \%$ to $2 \% " .5$ As for the gender differences, Schredl et al. ${ }^{11}$ stated that women tend to report nightmares more often than men but this gender difference was not found in children and older persons. In a similar vein, the DSM 5 stated that "adult females report having nightmares more frequently than do adult males. Nightmare content differs by sex, with adult females tending to report themes of sexual harassment or of loved ones disappearing/dying, and adult males tending to report themes of physical aggression or war/terror". Using Arab noninstitutionalized participants, female adolescents, undergraduates and employees reported nightmares more often than did their male counterparts. ${ }^{1-3,12}$ Several studies demonstrated significant relations between nightmares and psychopathology, especially neuroticism and anxiety. ${ }^{8,13-20}$ Ohayon et al. ${ }^{17}$ found a high rate of psychiatric disorders in an adult insomnia population with frequent nightmares. Persons having nightmare on a weekly basis were strongly related with depressed mood. ${ }^{21}$ Furthermore, there was a strong association between nightmares and border personality disorder. ${ }^{22}$ PTSD; $;^{10,23-25}$ and suicidality. ${ }^{26-31}$

Marinova et $a l .^{32}$ found that patients with recurrent depressive disorder suffered significantly more frequently from nightmares than those with bipolar disorder. Within the recurrent depressive disorder group, experiencing nightmares was associated with significantly higher scores on Hamiltion Depression Rating Scale suicide risk item, higher frequency of suicide attempts, and lower likelihood for lack of detectable suicide risk. They concluded that depressive patients suffering from nightmares showed significantly higher suicide risk. Unipolar depression appeared to be a stronger risk factor for suicidal behavior when accompanied with nightmares. Contrary to the lastmentioned results, some studies failed to find a correlation between nightmares and psychopathology, namely anxiety and neuroticism. ${ }^{33-37}$ Given the few Arabic studies on this domain, the aim of the present investigation was to explore the relation between reported nightmares and depressive symptoms. It was hypothesized that there will be positive correlations between nightmares and depression. The current investigation is unique because the vast majority of published researches in this field have been carried out on Western, Anglo-Saxon, and English-speaking participants. The Arab population is highly under-represented in the field of sleep medicine and psychopathology; however, culture may affect sleep disorders. Based on the results of the present research, cross-cultural comparisons would be feasible.

\section{Materials and methods}

\section{Participants}

A convenience sample of 8,218 school and university Arab students took part in this study ( $49 \%$ males). They were classified into 13 subgroups according to their ages from 12 to 24 years (Table 1). This sample was selected from the regular schools and different departments in The University. The researcher received approval to conduct the study from the Institutional Review Board. Informed consent was obtained from the guardians of children, whereas adults gave their oral approval to share as subjects in this study. The students did not receive any type of compensation for their participation in the study. It is important to note that the participants in this study were not diagnosed institutionalized patients. No exclusion criteria on psychiatric or neurological grounds were applied.

\section{Assessment tools}

Reported nightmares: A self-rating scale item was used to assess nightmares. ${ }^{1}$ The statement was as follows. "I have nightmares that wake me up scared". Participants were requested to respond to this item on a 5-point Likert- type scale as follows, 0: No,1: A little, 2: Modetrate, 3: Much and 4: Very much, so the possible range of scores is 0-4. Participants were instructed to answer this item, according to their subjective evaluation, on the basis of their perceived frequency during the past month, and not intensity. It is important to note that the concept of nightmare is quite clear to, and assimilated by, Arab participants' even young children in the present study (i.e., $12 \mathrm{yrs})$. The Arabic word to refer to this concept is well defined, i.e., "Kaboos". Unlike the "value neutral" English term, the "Kaboos" implies "pressure" or "stress", especially on the chest. ${ }^{1-2}$

The Center for Epidemiologic Studies-Depression Scale (CES-D). ${ }^{38}$ The CES-D scale is a 20 item self-report scale. It was developed to measure depressive symptomatology in adults in the general population. Items were selected from areas of depressive symptomatology previously described and validated. The items tap areas of depressed mood, feelings of guilt and worthlessness, feelings of helplessness and hopelessness, psychomotor retardation, loss of appetite and sleep disturbance. The scale items emphasize the affective component and depressed mood. ${ }^{39}$ Subjects respond to each item on a four-point scale according to the frequency of occurrence in the seven days previous to the testing. The original English from has been found to have very high internal consistency and adequate test-retest reliability. Construct validity has also been established. ${ }^{38}$ With the kind permission of the National Institute of Mental Health, the present researcher translated the CES-D scale into Arabic. Two bilingual $\mathrm{PhD}$ psychologists and two $\mathrm{PhD}$ linguists were requested to check the compatibility of meaning between the Arabic and English items of the scale. Suitable revisions and corrections were carried out. As a check on the adequacy of the English to Arabic translation, the back translation technique was applied. ${ }^{40-41} \mathrm{~A} \mathrm{PhD}$ linguist, who was unfamiliar with the scale, translated it back from the Arabic to English. Good results were achieved. Table 2 below presents the Cronbach's alpha and the one week test-retest reliabilities for the Arabic form. Criterion-related validity was 0.74 and 0.83 . Validity criteria were the SCL-90-D and the Hopkins Symptoms Check List-Depression Scale. 


\section{Procedure}

The Arabic versions of the nightmare item, along with the depression scale (CES-D) and other scales were administered to participants in group sessions of 30 to 35 students in their classrooms, during regular school and university hours. The scales were administered to each group in a single session of approximately 10 minutes in duration. Competent assistants carried out the administration of the tests. The university students provided verbal agreement to offer themselves as subjects, after the aims of the study were outlined. There were few refusals. As for the younger pupils, informed consent was obtained. Assurances were made that anonymity would be maintained. The response rate was $93 \%$. To compute the test-retest reliability, other participants were recruited (Table 2), and the interval between the test and retest was one week. The SPSS ${ }^{42}$ was used to compute the descriptive statistics, the $t$ ratios, effect size $d$, and the correlations between nightmares and depression.

\section{Results}

Table 1 sets out the descriptive statistics and the $t$ ratios for the nightmare rating scale among males and females. Reference to this table reveals that the sex-related differences were found only in the ages 15 to 21 in favor of females, and the effect size was small.

Table 2 sets out the reliabilities of the self-rating scale of nightmares based on a single item. Its 1 -week test-retest reliabilities ranged between 0.75 and 0.81 , indicating acceptable temporal stability and corroborate the trait (not state)-like of the score. The Cronbach alpha and retest reliabilities of the depression scale (CES-D) ranged between 0.71 and 0.84 , indicating acceptable to good internal consistency and temporal stability.

Table 3 presents the descriptive statistics and the t ratio for the depression scale in males and females. Inspection of this table indicates that the sex differences in depressive symptoms were statistically significant in nine out of the 13 age groups in favor of females, i.e., they reported more depressive symptoms. The effect size was small.

Table 4 sets out the Pearson correlation coefficients between nightmares and depressive symptoms in the ages 12 to 24 . Male and female samples in the same age group were combined because the differences between the correlations were trivial. Reference to this table indicated that all the correlations were statistically significant and positive. These correlations ranged from 0.295 to 0.619 and the median was 0.402 .

Table 1 Mean (M), standard deviation (SD), t-ratio, and the effect size $d$ for the self-rating scale item to assess nightmares in the past month among males and females in the ages 12-24

\begin{tabular}{|c|c|c|c|c|c|c|c|c|c|}
\hline \multirow{2}{*}{ Age } & \multicolumn{3}{|c|}{ Males } & \multicolumn{3}{|c|}{ Females } & \multirow{2}{*}{$\mathbf{t}$} & \multirow{2}{*}{ p } & \multirow{2}{*}{ d } \\
\hline & $\mathbf{N}$ & M & SD & $\mathbf{N}$ & $\mathbf{M}$ & SD & & & \\
\hline 12 & 267 & 0.94 & 1.29 & 231 & 0.73 & 1.14 & 1.89 & - & - \\
\hline 13 & 261 & 1.07 & 1.33 & 261 & 0.93 & 1.24 & 1.26 & - & - \\
\hline 14 & 351 & 0.84 & 1.11 & 454 & 0.96 & 1.17 & 1.41 & - & - \\
\hline 15 & 645 & 0.82 & 1.03 & 606 & 1.03 & 1.19 & 3.4 & 0.001 & 0.19 \\
\hline 16 & 654 & 0.79 & 1.05 & 570 & 1.11 & 1.23 & 4.85 & 0.0001 & 0.28 \\
\hline 17 & 502 & 0.74 & 0.98 & 576 & 1.06 & 1.18 & 4.81 & 0.0001 & 0.29 \\
\hline 18 & 450 & 0.72 & 0.93 & 578 & 1.06 & 1.13 & 5.16 & 0.0001 & 0.32 \\
\hline 19 & 330 & 0.8 & 0.95 & 265 & 1.13 & 1.12 & 3.82 & 0.0001 & 0.32 \\
\hline 20 & 219 & 0.89 & 1.02 & 235 & 1.14 & 1.13 & 2.43 & 0.02 & 0.23 \\
\hline 21 & 144 & 0.62 & 0.82 & 167 & 0.99 & 0.95 & 3.65 & 0.0001 & 0.42 \\
\hline 22 & 97 & 0.81 & 0.89 & 140 & 0.89 & 0.97 & 0.64 & - & - \\
\hline 23 & 62 & 0.85 & 0.96 & 69 & 0.96 & 0.98 & 0.6 & - & - \\
\hline 24 & 41 & 0.66 & 0.83 & 43 & 0.88 & 0.96 & 1.15 & - & - \\
\hline
\end{tabular}


Table 2 Reliability (r11) coefficients of the scales

\begin{tabular}{llll}
\hline Scales & r11 type & Group & r11 \\
\hline Nightmares & Test-retest** & children & 0.75 \\
- & - & adolescents & 0.8 \\
- & - & adults & 0.81 \\
CES-D* & Test-retest & males & 0.79 \\
- & - & females & 0.71 \\
- & Alpha & males & 0.8 \\
- & - & females & 0.84
\end{tabular}

* The center for epidemiologic studies-depression scale.

** The interval between the test and retest was one week.

Table 3 Mean (M), standard deviation (SD), t ratio, and the effect size $d$ for the depression scale in males and females

\begin{tabular}{|c|c|c|c|c|c|c|c|c|c|}
\hline \multirow{2}{*}{ Age } & \multicolumn{3}{|c|}{ Males } & \multicolumn{3}{|c|}{ Females } & \multirow{2}{*}{$\mathbf{t}$} & \multirow{2}{*}{$\mathbf{p}$} & \multirow{2}{*}{ d } \\
\hline & $\mathbf{N}$ & M & SD & $\mathbf{N}$ & $\mathbf{M}$ & SD & & & \\
\hline 12 & 267 & 16.33 & 12.63 & 231 & 17.7 & 12.39 & 0.24 & - & - \\
\hline 13 & 261 & 17.81 & 11.33 & 261 & 17.91 & 10.14 & 0.4 & - & - \\
\hline 14 & 351 & 16.84 & 9.11 & 454 & 19.35 & 11.62 & 3.29 & 0.001 & 0.23 \\
\hline 15 & 645 & 17.81 & 9.99 & 606 & 22.02 & 11.98 & 6.71 & 0.0001 & 0.38 \\
\hline 16 & 654 & 18.5 & 10.8 & 570 & 23.06 & 12.33 & 6.82 & 0.0001 & 0.39 \\
\hline 17 & 502 & 19.43 & 10.87 & 576 & 23.67 & 11.71 & 6.12 & 0.0001 & 0.37 \\
\hline 18 & 450 & 21.7 & 10.44 & 578 & 24.86 & 11.13 & 4.1 & 0.0001 & 0.26 \\
\hline 19 & 330 & 23.49 & 9.47 & 265 & 26.85 & 10.88 & 2.95 & 0.003 & 0.24 \\
\hline 20 & 219 & 22.91 & 10.06 & 235 & 25.3 & 10.61 & 1.58 & - & - \\
\hline 21 & 144 & 22.98 & 9.35 & 167 & 23.66 & 8.68 & 0.38 & - & - \\
\hline 22 & 97 & 20.56 & 7.49 & 140 & 24.97 & 9.75 & 2.17 & 0.03 & 0.29 \\
\hline 23 & 62 & 19.3 & 6.93 & 69 & 25.01 & 11.29 & 1.96 & 0.05 & 0.34 \\
\hline 24 & 41 & 17.01 & 8.1 & 43 & 24.94 & 10.03 & 2 & 0.05 & 0.44 \\
\hline
\end{tabular}

Table 4 The Pearson correlation coefficients (r) between nightmares and depression

\begin{tabular}{llll}
\hline Age & $\mathbf{N}$ & $\mathbf{r}$ & $\mathbf{p}$ \\
\hline 12 & 498 & 0.572 & 0.001 \\
13 & 522 & 0.47 & 0.001 \\
14 & 805 & 0.382 & 0.001 \\
15 & 1251 & 0.359 & 0.001 \\
16 & 1224 & 0.413 & 0.001 \\
17 & 1078 & 0.354 & 0.001 \\
18 & 1028 & 0.402 & 0.001 \\
19 & 595 & 0.419 & 0.001 \\
20 & 454 & 0.313 & 0.001 \\
21 & 311 & 0.295 & 0.001 \\
22 & 237 & 0.347 & 0.001 \\
23 & 131 & 0.458 & 0.001 \\
24 & 84 & 0.619 & 0.001 \\
Median & 8218 & 0.402 & 0.001 \\
\hline
\end{tabular}




\section{Discussion}

The main thesis of the present investigation has been successfully demonstrated. That is, the correlations between reported nightmares and depressive symptoms were statistically significant and positive among the 13 age groups of school and university students (from 12 to 24 years) (Table 4). These correlations ranged between 0.3 and 0.6 , with a median of 0.4 . The present results are congruent with many previous findings correlating nightmares with different types of psychopathology, including depression (see the introduction section). As stated by Levin and Fireman, ${ }^{16}$ the experience of frequent idiopathic nightmares was associated with significantly higher scores on almost all measures of psychological disturbance. The correlations between nightmares and depressive symptoms mean co-variation of the two constructs. However, the correlation, ipso facto, did not mean causality. That is, nightmare is not the cause of depressive symptoms and vise versa. To address causality, this needs another methodology, either experimental design or regression analysis. Some studies explored the link between nightmares and psychopathology, namely anxiety and depression. ${ }^{6,16}$ Results strongly supported the view that nightmares are related to social and psychological problems while awake. It could be suggested that these problems and hardship may affect both nightmares' frequency and depression level. Regarding the sex-related differences in nightmares (Table 1), females obtained higher mean scores than did their male counterparts in all the 13 comparisons from age 12 to 24 . However, the statistically significant differences were only found in seven age groups (from 15 to 21 years). This gender difference was consistent with previous findings. ${ }^{1,11}$ As for the depression level (Table 3), females obtained higher mean scores than did their male peers in all the 13 age groups. However, the statistically significant differences in depression were found only in nine age groups, in which females had the higher mean scores. This result is consistent with previous studies. ${ }^{43}$

\section{Limitation}

Despite the large sample size from an under-studied population in the field of sleep disorders, and the good psychometric properties of the assessment scales, specific limitations have to be acknowledged. Foremost among them is the selection of a convenience sample and not a random or probability sample. Further, student samples have specific characteristics in age range, education, and intelligence. They represent a small sector of any given society. Thus, the generalization of the present results needs further studies. Therefore, it would be suitable to replicate the present study using a probability sample in another age group, e.g., the late adulthood or the old age. It is also recommended to assess the incidence, frequency, and distress associated with the nightmare experience as well as the association between nightmares and insomnia

\section{Conclusion}

Based on the characteristics of both the sample and the assessment scales in the present study on Arab participants, the salient finding is the statistically significant and positive association between reported nightmares and depressive symptoms in all the 13 age groups from 12 to 24 years. This result means that persons experiencing frequent nightmares report themselves as having high depressive symptoms. Thus, the depression score should be assessed in persons experiencing nightmares. Future research with clinical cases would investigate the following hypothesis: treating nightmares would reduce the score of depression and ameliorate the level of happiness and adjustment of the person.

\section{Acknowledgements}

I thank the participants, the research assistants, the reviewers of the manuscript, and the editorial board of this periodical.

\section{Conflict of interest}

The author declares no Conflict of interest.

\section{References}

1. Abdel-Khalek AM. Nightmares: prevalence, age and gender differences among Kuwaiti children and adolescents. Sleep and Hypnosis. 2006;8:3340 .

2. Abdel-Khalek AM. Prevalence rates of reported nightmares in a cross sectional sample of Kuwaiti children, adolescents, undergraduates and employees. Sleep and Hypnosis. 2010;12(1-2):13-22.

3. Abdel-Khalek AM. Reported nightmares and trait anxiety among Arab children, adolescents and adults. Journal of Sleep Disorders \& Therapy. 2016;5:4.

4. American academy of sleep medicine. International classification of sleep disorders, revised: diagnostic and coding manual. Chicago, Illinois, USA; 2001.

5. American psychiatric association. Diagnostic and Statistical Manual of Mental Disorders. 5th ed. Arlington, USA: APA; 2013.

6. Zadra A, Donderi DC. Nightmares and bad dreams: Their prevalence and relationship to well-being. Journal J Abnorm Psychol. 2000;109(2):273281.

7. Silvestri R, Arico I. Parasomnias. In HR Smith, et al, editors. Sleep medicine. New York, USA: Cambridge University Press; 2008. p. 47-55.

8. WHO. International statistical classification of diseases and related health problems. $10^{\text {th }}$ revision. (ICD-10) 2nd ed. Geneva: World Health Organization; 2005.

9. Hartman E. Nightmares and other dreams. In: MH Kryger, et al, editors. Principles and practice of sleep medicine. Saunders, Philadelphia, USA; 1994. p. 407-410.

10. Shneerson JM. Sleep medicine: a guide to sleep and its disorders. 2nd ed. UK: Oxford; 2005.

11. Schredl M, Reinhard I. Gender differences in nightmare frequency: a metaanalysis. Sleep Med Rev. 2011;15(2):115-121.

12. Abdel-Khalek AM. Epidemiologic study of sleep disorders in Kuwaiti adolescents. Perceptual and Motor Skills. 2001;93(3):901-910.

13. Berquier A, Ashton R. Characteristics of the frequent nightmare sufferer. $J$ Abnorm Psychol. 1992;101(2):246-250.

14. Köthe M, Pietrowsky R. Behavioral effects of nightmares and their correlations to personality patterns. Dreaming. 2001;11(1):43-52.

15. Lang R, O'Connor KP. Personality, dream content, and dream coping style. Personality and Individual Differences. 1984;5(2):211-219.

16. Levin R, Fireman G. Nightmare prevalence, nightmare distress, and selfreported psychological disturbance. Sleep. 2002;25(2):205-212.

17. Ohayon MM, Morselli PL, Guilleminault C. Prevalence of nightmares and their relationship to psychopathology and daytime functioning in insomnia subjects. Sleep. 1997;20(5):340-348. 
18. Roberts J, Lennings CL. Peronality, psychopathology and nightmares in young people. Personality and Individual Differences. 2006;41(4):733744

19. Schredl M, Landgraf C, Zeiler O. Nightmare frequency, nightmare distress and neuroticism. North American Journal of Psychology. 2003;5(3):345350 .

20. Spoormaker VI, Schredl M, Van den Bout J. Nightmares: from anxiety symptom to sleep disorder. Sleep Med Rev. 2006;10(1):19-31.

21. Miro E, Martinez MP. Affective and personality characteristics in function of nightmare prevalence, nightmare distress, and interference due to nightmares. Dreaming. 2005;15:89-105.

22. Semiz U, Basoglu C, Ebrinc S, et al. Nightmare disorder, dream anxiety, and subjective sleep quality in patients with borderline personality disorder. Psychiatry Clin Neurosci. 2007;62(1):48-55.

23. Davis JL, Byrd P, Rhudy JL, et al. Characteristics of chronic nightmares in a trauma-exposed treatment-seeking sample. Dreaming. 2007;17(4):187198.

24. Germain A, Nielsen TA. Sleep pathophysiology in posttraumatic stress disorder and idiopathic nightmare sufferers. Biol Psychiatry. 2003;54(10):1092-1098.

25. Pagel J F. Nightmares and disorders of dreaming. Am Fam Physician. 2000;61(7):2037-2042.

26. Agargun MY, Besiroglu L, Cilli AS, et al. Nightmares, suicide attempts, and melancholic features in patients with major depression. J Affect Diord. 2007;98(3):267-270

27. Agargun MY, Cilli AS, Kara H, et al. Repetitive and frightening dreams and suicidal behavior in patients with major depression. Compr Psychiatry. 1998;39(4):198-202.

28. Ağargün MY, Kara H, Solmaz M. Sleep disturbances and suicidal behavior in patients with major depression. I Clin Psychiatr. 1997;58(6):249-251.

29. Agargun MY, Kara H, Ozer OA, et al. Clinical importance of nightmare disorder in patients with dissociative disorders. Psychiatry Clin Neurosciences. 2003;57(6):575-579.

30. Sjostrom N, Weam M, Hetta J. Nightmares and sleep disturbances in relation to suicidality in suicide attempters. Sleep. 2007;30(1):91-95.
31. Tanskanen A, Tuomilehto J, Viinamaki H, et al. Nightmares as predictors of suicide. Sleep. 2001;24(7):844-847.

32. Marinova P, Koychev I, Laleva L, et al. Nightmares and suicide: Predicting risk in depression. Psychiatr Danub. 2014;26(2):159-164.

33. Belicki K. Nightmare frequency versus nightmare distress: relations to psychopathology and cognitive style. Journal of Abnormal Psychology. 1992;101(3):592-597.

34. Beullens J. Nightmares in psychiatry. Acta Neuropsychiatr. 1996;8(1):1216.

35. Chivers L, Blagrove M. Nightmare frequency, personality and acute psychopathology. Personality and Individual Differences. 1999;27(5):843851.

36. Hartman E, Russ D, Oldfield M, et al. Who has nightmares? The personality of the lifelong nightmare sufferer. Arch Gen Psychiatry. 1987;44(1):49-56.

37. Wood J, Bootzin R. The prevalence of nightmares and their independence from anxiety. J Abnorm Psychol. 1990;99(1):64-68.

38. Radloff LS. The CES-D Scale: A self-report depression scale for research in the general population. Applied Psychological Measurement. 1997;1(3):385-401.

39. Katz R, Shaw BF, Vallis TM, et al. The assessment of severity and symptom patterns in depression. In EE Beckham, et al, ediors. Handbook of Depression. 2nd ed. New York, NY, USA: Guilford Press; 1995.

40. Brislin RW. Back translation for cross-cultural research. Journal of CrossCultural Psychology. 1970;1(3):185-216.

41. Brislin RW. Translation and content analysis of oral and written material. In HC Triandis \& JW Berry (Eds.), Boston: Allyn \& Bacon, USA. Handbook of Cross-cultural psychology. 1980;2:389-444

42. SPSS. Statistical data analysis: Base 18.0, Users guide. Chicago, Illinois USA: SPSS; 2009.

43. Nolen-Hoeksema S. Gender differences in depression. IH Gotlib, et al, editors. Handbook of depression. New York: USA: Guilford; 2002. 